\title{
Nonlinear State Space Modeling and System Identification for Electrohydraulic Control
}

\author{
Jun Yan, Bo Li, Hai-Feng Ling, Hai-Song Chen, and Mei-Jun Zhang \\ College of Field Engineering, PLA University of Science and Technology, Nanjing 210007, China \\ Correspondence should be addressed to Hai-Feng Ling; hf.ling@ymail.com
}

Received 6 January 2013; Accepted 29 January 2013

Academic Editor: Shengyong Chen

Copyright ( 2013 Jun Yan et al. This is an open access article distributed under the Creative Commons Attribution License, which permits unrestricted use, distribution, and reproduction in any medium, provided the original work is properly cited.

\begin{abstract}
The paper deals with nonlinear modeling and identification of an electrohydraulic control system for improving its tracking performance. We build the nonlinear state space model for analyzing the highly nonlinear system and then develop a HammersteinWiener (H-W) model which consists of a static input nonlinear block with two-segment polynomial nonlinearities, a linear timeinvariant dynamic block, and a static output nonlinear block with single polynomial nonlinearity to describe it. We simplify the $\mathrm{H}-\mathrm{W}$ model into a linear-in-parameters structure by using the key term separation principle and then use a modified recursive least square method with iterative estimation of internal variables to identify all the unknown parameters simultaneously. It is found that the proposed H-W model approximates the actual system better than the independent Hammerstein, Wiener, and ARX models. The prediction error of the H-W model is about $13 \%, 54 \%$, and $58 \%$ less than the Hammerstein, Wiener, and ARX models, respectively.
\end{abstract}

\section{Introduction}

Electrohydraulic control systems are widely used in industry, due to their unique features of small size to power ratio, high nature frequency, high position stiffness, and low position error [1]. However, the dynamics of hydraulic systems is highly nonlinear in nature. The systems may be subjected to nonsmooth nonlinearities due to control input saturation, friction, valve overlapping, and directional changes of valve opening. A number of robust and adaptive control strategies have been proposed to deal with such problems [2-4], but modeling and identification of control systems remain an important and difficult issue in most real-world applications.

Linear models of electrohydraulic control systems are simple and widely used, but they assume that the hydraulic actuator always moves around an operating point $[5,6]$, which does not accord with most real-world cases where the actuator moves in a wide range with hard nonlinearities. In the literature, Wang et al. [7] analyzed the nonlinear dynamic characteristics of hydraulic cylinder, such as nonlinear gain, nonlinear spring, and nonlinear friction force. Jelali and Schwarz [8] identified the nonlinear models in observer canonical form of hydraulic servodrives. Kleinsteuber and Sepehri [9] used a polynomial abductive network modeling technique to describe a class of hydraulic actuation systems which were used in heavy-duty mobile machines. Yousefi et al. [10] proposed the Differential Evolution algorithm to identify the nonlinear model of a servohydraulic system with flexible load. Yao et al. [2] also pointed out that there were many considerable model uncertainties, such as parametric uncertainties and uncertain nonlinearities. As we can see, modeling and identifying the electrohydraulic control system as a flexible nonlinear black-box or grey-box are more appropriate for real-world applications.

In the field of nonlinear system identification, the Hammerstein and Wiener (H-W) models are widely used [11]. Kwak et al. [12] proposed two Hammerstein-type models to identify hydraulic actuator friction dynamics. The Hammerstein-type models are built by linear time-invariant (LTI) dynamic subsystems and static nonlinear (SN) elements in a cascade structure; they are able to approximate most of the nonlinear dynamics with an arbitrarily high accuracy, and can generate both physical insights and flexible structures. Generally, the Wiener model is supposed to represent the output nonlinearities and sensor nonlinearities, while the Hammerstein model is supposed to represent the input nonlinearities and actuator nonlinearities. The HammersteinWiener $(\mathrm{H}-\mathrm{W})$ model, which is defined as a static nonlinear 


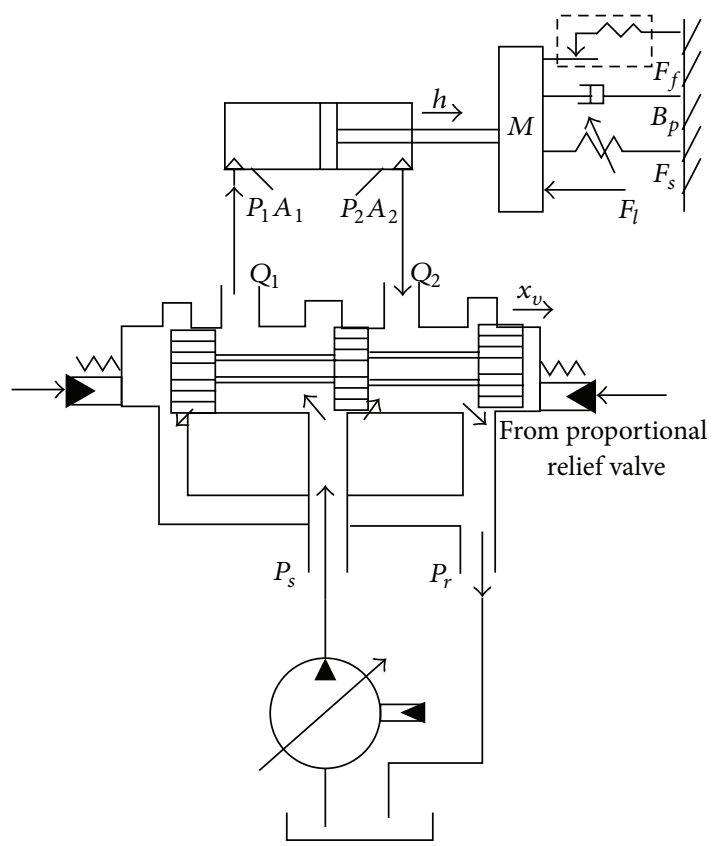

FIGURE 1: Valve controlled asymmetric cylinder system.

element in cascade with a linear dynamic system followed by another static nonlinear element, is adopted in this paper.

The H-W model is a parameterized nonlinear model in black-box term. There are two advantages of the H-W model. The first one is that only the input and output singles are used for identification of all the unknown parameters; that is, no information on the internal states is needed, which can simplify the identification process and improve the prediction accuracy by less sensors and noise. The second one is that it has a physical insight into the nonlinear characteristics of the actual system, which is important in system analyzing, monitoring, diagnosis, and controller design.

The rest of this paper is organized as follows. Section 2 presents the theoretic modeling of an electrohydraulic control system. Section 3 describes our H-W model in detail. Section 4 proposes the iterative identification algorithm for the H-W model. Section 5 presents the experimental tests as well as the identification results. Finally, Section 6 concludes the paper.

\section{Theoretic Modeling}

A general electrohydraulic control system is mainly comprised of an electrohydraulic proportional valve and a valve controlled asymmetric cylinder. In this paper, we study a proportional relief valve controlled valve-cylinder system as shown in Figure 1, where $h$ is the displacement of piston, $M$ is the equivalent load mass, $A_{1}$ and $A_{2}$ are the areas of piston in the head and rod sides of cylinder, $P_{1}$ and $P_{2}$ are the pressures inside the two chambers of the cylinder, $P_{s}$ is the supply pressure, $P_{r}$ is the pressure of return oil, $Q_{1}$ and $Q_{2}$ are the flows in and out of the cylinder, $x_{v}$ is displacement of the spool valve, $B_{p}$ is the viscous damping coefficient, $F_{f}$ represents nonlinear friction, $F_{s}$ represents nonlinear spring

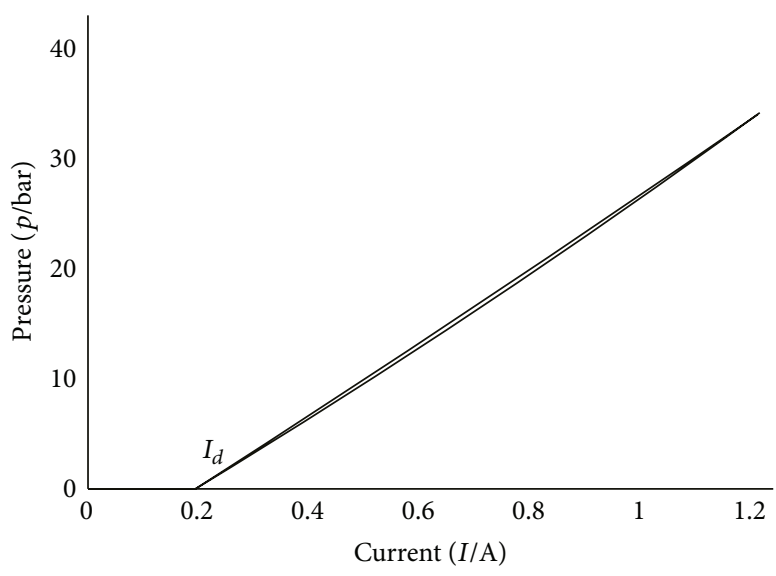

(a) Dead band of the pilot relief valve

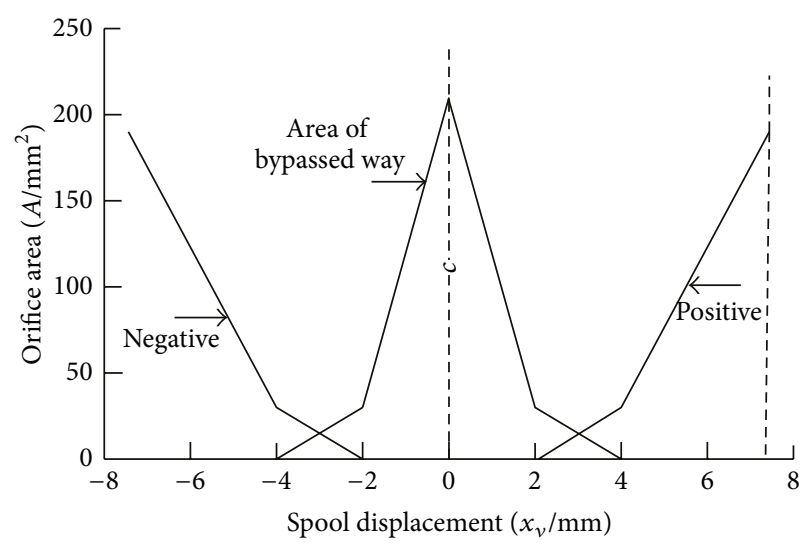

(b) Dead band of the main valve

Figure 2: Dead band of the electrohydraulic proportional system.

force, $F_{c}$ represents viscous force, and $F_{l}$ represents uncertain load.

Modeling the system by physical laws gives us a particular insight into the system's properties, which allows us to seek the parameterized models that are flexible enough to capture all dynamic behavior of the system [13, 14]. The electrohydraulic proportional valve is controlled directly by the digital controller. It can be modeled as a first order transfer function [9]:

$$
G_{l}=\frac{x_{v}(s)}{I_{v}(s)}=\frac{k_{v}}{1+\tau_{v} s},
$$

where $k_{v}$ is the gain of the electrohydraulic proportional valve, $\tau_{v}$ is the time constant of the first order system, $I_{v}=I-I_{d}$ is the effective current, $I$ and $I_{d}$ are the practical input current of the proportional relief valve and the current to overcome dead band of the valve, respectively. The dead bands mainly due to the pilot relief valve and the main valve are depicted in Figure 2.

The valve controlled asymmetric cylinder is shown in Figure 1. Generally, its model is constructed by combining the flow equation of spool valve, the continuity equation 
of hydraulic cylinder, and the force equilibrium equation of hydraulic cylinder [2]. Define the state variables as

$$
\left[\begin{array}{lllll}
x_{1} & x_{2} & x_{3} & x_{4} & x_{5}
\end{array}\right]^{T} \triangleq\left[\begin{array}{lllll}
h & \dot{h} & p_{1} & p_{2} & x_{v}
\end{array}\right]^{T} .
$$

The entire system can be modeled as the following nonlinear state space model [15]:

$$
\begin{gathered}
\dot{x}_{1}=x_{2}, \\
\dot{x}_{2}=\frac{A_{1} x_{3}}{M}-\frac{A_{2} x_{4}}{M}-\frac{F_{f}\left(x_{2}\right)}{M} \\
-\frac{F_{s}\left(x_{1}\right)}{M}-\frac{F_{c}\left(x_{2}\right)}{M}-\frac{F_{l}}{M}, \\
\dot{x}_{3}=-\frac{\beta_{e} A_{1} x_{2}}{V_{1}}-\frac{\beta_{e}\left(C_{i}+C_{e}\right) x_{3}}{V_{1}}+\frac{\beta_{e} C_{i} x_{4}}{V_{1}}+\frac{\beta_{e} g_{1}(x)}{V_{1}} x_{5}, \\
\dot{x}_{4}=\frac{\beta_{e} A_{2} x_{2}}{V_{2}}+\frac{\beta_{e} C_{i} x_{3}}{V_{2}}-\frac{\beta_{e}\left(C_{i}+C_{e}\right) x_{4}}{V_{2}}-\frac{\beta_{e} g_{2}(x)}{V_{2}} x_{5}, \\
\dot{x}_{5}=\frac{k_{v}}{\tau_{v}} I_{v}-\frac{x_{5}}{\tau_{v}}, \\
\times C_{d 1} W \sqrt{\frac{2}{\rho}\left(\left(1+\operatorname{sgn}\left(x_{5}\right)\right) \frac{p_{s}}{2}-\operatorname{sgn}\left(x_{5}\right) x_{3}\right)}, \\
g_{2}(x)=\operatorname{sgn}\left(\left(1-\operatorname{sgn}\left(x_{5}\right)\right) \frac{p_{s}}{2}+\operatorname{sgn}\left(x_{5}\right) x_{4}\right) \\
\times C_{d 2} W \sqrt{\frac{2}{\rho}\left(\left(1-\operatorname{sgn}\left(x_{5}\right)\right) \frac{p_{s}}{2}+\operatorname{sgn}\left(x_{5}\right) x_{4}\right)},
\end{gathered}
$$

where $\beta_{e}$ is the effective bulk modulus, $V_{1}$ and $V_{2}$ are effective volumes of the two chambers, $C_{i}$ and $C_{e}$ are internal and external leakage coefficients, $W$ is the area gradient of the valve orifice, and $C_{d 1}$ and $C_{d 2}$ are flow discharge coefficients of the spool valve.

Several physical phenomena have been taken into consideration in the above model, for example, nonlinear friction $F_{f}$, nonlinear spring force $F_{s}$, viscous force $F_{c}$, uncertain load $F_{l}$, discontinuous flow discharge $g_{i}$, oil compliance, internal leakage, and external leakage. From the theoretic modeling of the electrohydraulic control system, we can see that the system is a highly nonlinear system containing complex features, such as the dead band nonlinearity, saturation, squared pressure drop, and asymmetric response property.

There are also some hard-to-model nonlinearities in (3), such as nonlinear friction, nonlinear spring force, and uncertain external disturbances. So, modeling this system just by physical laws fails to approximate the actual system. Furthermore, identification of the unknown parameters in (3) is hard due to its demand on internal states measurement. In the following, we adopt an $\mathrm{H}-\mathrm{W}$ model to model this highly

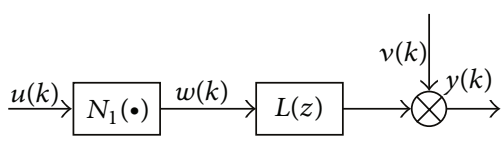

(a) Hammerstein model

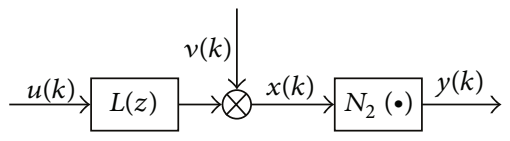

(b) Wiener model

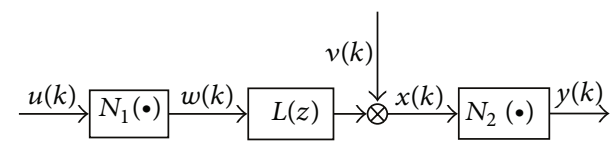

(c) Hammerstein-Wiener model

Figure 3: Hammerstein and Wiener models.

nonlinear dynamic system. The $\mathrm{H}-\mathrm{W}$ model is a flexible black-box model based on the physical insight into the actual system. We identify the parameters of the H-W model using the input and output signals, which can simplify the identification process and improve the prediction accuracy by less sensors and noise.

\section{Hammerstein-Wiener Model}

The "universal" nonlinear black-box methods, such as neural networks, Volterra series, and fuzzy models, are widely used to model complex nonlinear systems. Most of these methods can avoid unmodeled dynamics in the aforementioned mathematical model $[16,17]$. However, these models do not provide deep insight into the nonlinear characteristics of the actual system, which is important in system analyzing, monitoring, diagnosis, and controller design. In comparison, the Hammerstein-Wiener (H-W) model possesses the flexibility to capture all relevant nonlinear phenomena as well as the physical insight into the actual system. In this section, we develop an H-W model to describe the electrohydraulic control system.

The H-W model is composed of an internal linear dynamic block and two static nonlinear blocks; it is the combination of Hammerstein and Wiener model. The Hammerstein model is a nonlinear model with a static nonlinear block followed by a linear dynamic block, as shown in Figure 3(a), and this N-L type of model may account for actuator nonlinearities and other input nonlinear effects. The Wiener model has linear dynamic block followed by a nonlinear block, as shown in Figure 3(b), and this L-N type of model mainly accounts for sensor nonlinearities and output nonlinear effects. A series combination of a Hammerstein and a Wiener model yields the H-W model, as shown in Figure 3(c), and this N-L-N type of model has both characteristics of the Hammerstein and Wiener models. Moreover, all of the three models have proved to be able to accurately describe a wide variety of nonlinear systems in [9].

According to the nonlinearities of the abovementioned electrohydraulic control system, for example, dead band, 
saturation, nonlinear friction, nonlinear spring force, and asymmetric dynamics of the cylinder, we describe the input nonlinearity $\left(N_{1}\right)$ block of the models in Figure 3 by a two-segment polynomial nonlinearities. The two-segment polynomial nonlinearities have the advantage of describing a system whose dynamic properties differ significantly at the positive and negative directions [18]; it has less parameters to be estimated than a single polynomial and piecewise linear models [19]. It can be written as

$$
w(k)= \begin{cases}f(u(k))=\sum_{l=0}^{r_{1}} f_{l} u^{l}(k), & u(k) \geq 0, \\ g(u(k))=\sum_{l=0}^{r_{1}} g_{l} u^{l}(k), & u(k)<0,\end{cases}
$$

where $f_{l}$ and $g_{l}$ are parameters of the polynomial function, $u(k)$ is the input, $w(k)$ is the output of static nonlinear function $N_{1}$, and $r_{1}$ is the degree of the polynomial function.

Define the switching function as

$$
h(u)= \begin{cases}0, & u \geq 0 \\ 1, & u<0\end{cases}
$$

Then the relation between inputs $\{u(k)\}$ and outputs $\{w(k)\}$ of the input nonlinear block can be written as

$$
\begin{aligned}
w(k) & =f(u(k))+(g(u(k))-f(u(k))) h(u(k)) \\
& =\sum_{l=0}^{r_{1}} f_{l} u^{l}(k)+\sum_{l=0}^{r_{1}} p_{l} u^{l}(k) h(u(k)),
\end{aligned}
$$

where $p_{l}=g_{l}-f_{l}$.

The difference equation model $L(z)$ of the linear dynamic block is described by an extended autoregressive (ARX) model as

$$
A\left(z^{-1}\right) x(k)=z^{-n_{k}} B\left(z^{-1}\right) w(k)+v(k)
$$

where $w(k)$ and $x(k)$ are the input and output of the linear dynamic block, respectively, $v(k)$ is white noise, $n_{k}$ represents the pure delay of the system, and $A\left(z^{-1}\right)$ and $B\left(z^{-1}\right)$ are scalar polynomials in the unit delay operator $z^{-1}$ :

$$
\begin{aligned}
& A\left(z^{-1}\right)=1+a_{1} z^{-1}+\cdots+a_{n_{a}} z^{-n_{a}}, \\
& B\left(z^{-1}\right)=b_{0}+b_{1} z^{-1}+\cdots+b_{n_{b}} z^{-n_{b}} .
\end{aligned}
$$

The output nonlinear block $N_{2}$ is described by a single polynomials:

$$
y(k)=q(x(k))=\sum_{m=1}^{r_{2}} q_{m} x^{m}(k),
$$

where $q_{m}$ is unknown parameter, $r_{2}$ is the degree of the polynomial function $N_{2}$, and $y(k)$ is output of the entire system, and in this paper, it represents the output velocity.

The H-W model of the system is depicted in Figure 4.

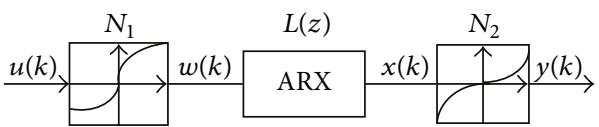

FIGURE 4: Schematic diagram of the H-W model.

\section{Iterative Identification Algorithm}

As we know, the cascade mode of the models depicted in Figure 3 leads to composite mappings, for example, Hammerstein model: $L\left(N_{1}(u(k))\right)$, Wiener model: $N_{2}(L(u(k)))$, H-W model: $N_{2}\left(L\left(N_{1}(u(k))\right)\right)$. Substituting the mathematic models of each block (i.e., (7), (8), and (10)) into the composite mappings directly leads to complex models which are strongly nonlinear in both of the variables and the unknown parameters. It is not appropriate for parameter estimation [20]. In the following, we apply the so-called key term separation principle to simplify the $\mathrm{H}-\mathrm{W}$ model into a linearin-parameters structure and then adopt a modified recursive least square algorithm with internal variable estimation to estimate both of the linear and nonlinear block parameters simultaneously.

4.1. Key Term Separation Principle. Let $f, g$, and $h$ be one-toone mappings defined on nonempty sets $U, X$, and $Y$ as

$$
\begin{gathered}
f: U \longrightarrow X, \\
g: X \longrightarrow Y, \\
h=g \circ f: U \longrightarrow Y .
\end{gathered}
$$

Then the composite mapping $h$ can be given by

$$
y(t)=g[x(t)]=g[f[u(t)]]=h[u(t)] .
$$

The basic idea of key term separation principle is a form of half-substitution suggested in [21]. Suppose $g$ be an analytic nonlinear mapping which can be rewritten into the following additive form:

$$
y(t)=x(t)+G[x(t)]
$$

Which consists of the key term $x(t)$, plus the remainder of the original mapping assigned as $G(\cdot)$. Rewrite the one-to-one mapping $f$

$$
x(t)=f[u(t)] .
$$

We substitute (13) only into the first term in the right side of (14) and then obtain the following mapping:

$$
y(t)=f[u(t)]+G[x(t)] .
$$

Equations (14) and (15) describe the mapping function $h$ in a compositional way. This makes the inner mapping appears both explicitly and implicitly in the outer one, which may be helpful for parameter identification. Note that, this decomposition technique can easily be extended to a more multilayer composite mapping. 
4.2. Modified Least Square Algorithm. In this section, we decompose the H-W model into a linear-in-parameters structure by the key term separation principle and develop a modified iterative least square algorithm with internal variables estimation to identify all the unknown parameters of the H-W model. We also apply this method to the Hammerstein and Wiener models.

According to the key term separation principle, we rewrite the output nonlinear block $N_{2}$, that is, (10) as

$$
y(k)=q_{1} x(k)+\sum_{m=2}^{r_{2}} q_{m} x^{m}(k)
$$

where the internal variable $x(k)$ is separated. The dynamic linear block $L(z)$, that is, (8) can be rewritten as

$$
\begin{aligned}
x(k)= & b_{0} w(k) z^{-n_{k}}+z^{-n_{k}}\left[B\left(z^{-1}\right)-b_{0}\right] w(k) \\
& +\left[1-A\left(z^{-1}\right)\right] x(k),
\end{aligned}
$$

where the internal variable $w(k)$ is separated. Now, to complete the sequential decomposition, first, we substitute (7) into (17) only for $w(k)$ in the first term and then substitute the new equation (17) into (16) only for $x(k)$ in the first term again. The final output equation of the $\mathrm{H}-\mathrm{W}$ model will be

$$
\begin{aligned}
y(k)=q_{1}\left\{b_{0}\right. & (f(u(k))+p(u(k)) h(u(k))) z^{-n_{k}} \\
+ & z^{-n_{k}}\left[B\left(z^{-1}\right)-b_{0}\right] w(k) \\
+ & {\left.\left[1-A\left(z^{-1}\right)\right] x(k)\right\}+\sum_{m=2}^{r_{2}} q_{m} x^{m}(k) . }
\end{aligned}
$$

As the H-W model depicted in Figure 4 consists of three subsystems in series, the parameterization of the model is not unique because many combinations of parameters can be found [22]. Therefore, one parameter in at least two blocks has to be fixed in (18). Evidently, the choices $q_{1}=1$ and $b_{0}=1$ will simplify the model description. Then, the H-W model can be written as

$$
\begin{aligned}
y(k)= & \sum_{l=0}^{r_{1}} f_{l} u^{l}\left(k-n_{k}\right)+\sum_{l=0}^{r_{1}} p_{l} u^{l}\left(k-n_{k}\right) h\left(u\left(k-n_{k}\right)\right) \\
& +\sum_{i=1}^{n_{b}} b_{i} w\left(k-n_{k}-i\right)+\sum_{j=1}^{n_{a}} a_{j} x(k-j)+\sum_{m=2}^{r_{2}} q_{m} x^{m}(k) .
\end{aligned}
$$

Equation (19) is linear-in-parameters for given $u(k), x(k)$, and $w(k)$; it can be written in the following least square format:

$$
y(k)=\Phi^{T}(k, \theta) \theta
$$

where the internal variables $w(k)$ and $x(k)$ are estimated by (7) and (17) using the preceding estimated parameters during each iterative process and

$$
\begin{aligned}
\Phi^{T}= & {\left[1, u\left(k-n_{k}\right), \ldots, u^{r_{1}}\left(k-n_{k}\right),\right.} \\
& h\left(u\left(k-n_{k}\right)\right), u\left(k-n_{k}\right) h\left(u\left(k-n_{k}\right)\right), \ldots, \\
& u^{r_{1}}\left(k-n_{k}\right) h\left(u\left(k-n_{k}\right)\right), w\left(k-n_{k}-1\right), \ldots, \\
& w\left(k-n_{k}-i\right),-x(k-1), \ldots,-x(k-j), \\
& \left.x^{2}(k), \ldots, x^{r_{2}}(k)\right], \\
\theta= & {\left[f_{0}, f_{1}, \ldots, f_{r_{1}}, p_{0}, p_{1}, \ldots, p_{r_{1}}, b_{1}, \ldots, b_{n_{b}},\right.} \\
& \left.a_{1}, \ldots, a_{n_{a}}, q_{2}, \ldots, q_{r_{2}}\right] .
\end{aligned}
$$

Now, we apply the modified recursive least square method with iterative estimation of the internal variable to (20) [17]. Minimizing the following least square criterion [12]:

$$
\widehat{\theta}=\underset{\theta}{\arg \min } \sum_{k=1}^{n} \lambda^{n-k}\left[y(k)-\widehat{\Phi}^{T}(k) \theta\right],
$$

where $\lambda \leqq 1$ is the forgetting factor, the formulas of the recursive identification algorithm supplemented with internal variable estimation are as follows:

$$
\widehat{\theta}(k)=\widehat{\theta}(k-1)+\frac{\mathbf{P}(k-1) \widehat{\Phi}(k)\left[y(k)-\widehat{\Phi}^{T}(k) \widehat{\theta}(k-1)\right]}{\lambda+\widehat{\Phi}^{T}(k) \mathbf{P}(k-1) \widehat{\Phi}(k)},
$$

$$
\begin{aligned}
& \mathbf{P}(k)=\frac{\mathbf{P}(k-1)}{\lambda}-\frac{\mathbf{P}(k-1) \widehat{\Phi}(k) \widehat{\Phi}^{T}(k) \mathbf{P}(k-1)}{1+\widehat{\Phi}^{T}(k) \mathbf{P}(k-1) \widehat{\Phi}(k) / \lambda}, \\
& \widehat{w}(k)=\sum_{l=0}^{r_{1}} \widehat{f}_{l}(k-1) u^{l}(k)+\sum_{l=0}^{r_{1}} \widehat{p}_{l}(k-1) u^{l}(k) h(u(k)),
\end{aligned}
$$

$$
\begin{aligned}
\widehat{x}(k)= & \widehat{w}\left(k-n_{k}\right)+\sum_{i=1}^{n_{b}} \widehat{b}_{i}(k-1) \widehat{w}\left(k-n_{k}-i\right) \\
& -\sum_{j=1}^{n_{a}} \widehat{a}_{j}(k-1) x(k-j),
\end{aligned}
$$

$$
\begin{aligned}
\widehat{\Phi}(k)=[ & 1, u\left(k-n_{k}\right), \ldots, u^{r_{1}}\left(k-n_{k}\right), h\left(u\left(k-n_{k}\right)\right), \\
& u\left(k-n_{k}\right) h\left(u\left(k-n_{k}\right)\right), \ldots, \\
& u^{r_{1}}\left(k-n_{k}\right) h\left(u\left(k-n_{k}\right)\right), \\
& \widehat{w}\left(k-n_{k}-1\right), \ldots, \widehat{w}\left(k-n_{k}-i\right), \\
& \left.-\widehat{x}(k-1), \ldots,-\widehat{x}(k-j), \widehat{x}^{2}(k), \ldots, \widehat{x}^{r_{2}}(k)\right]^{T},
\end{aligned}
$$

where $\mathbf{P}(0)=\mu \mathbf{I}$, I is unit matrix, and $0<\mu<\infty$. 


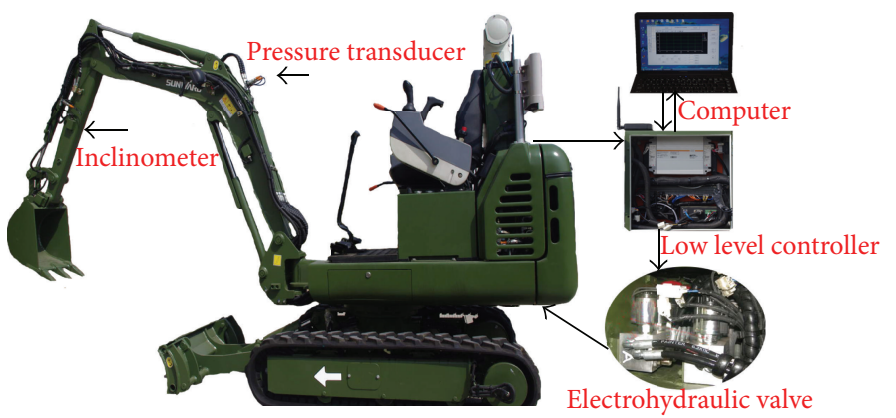

(a)

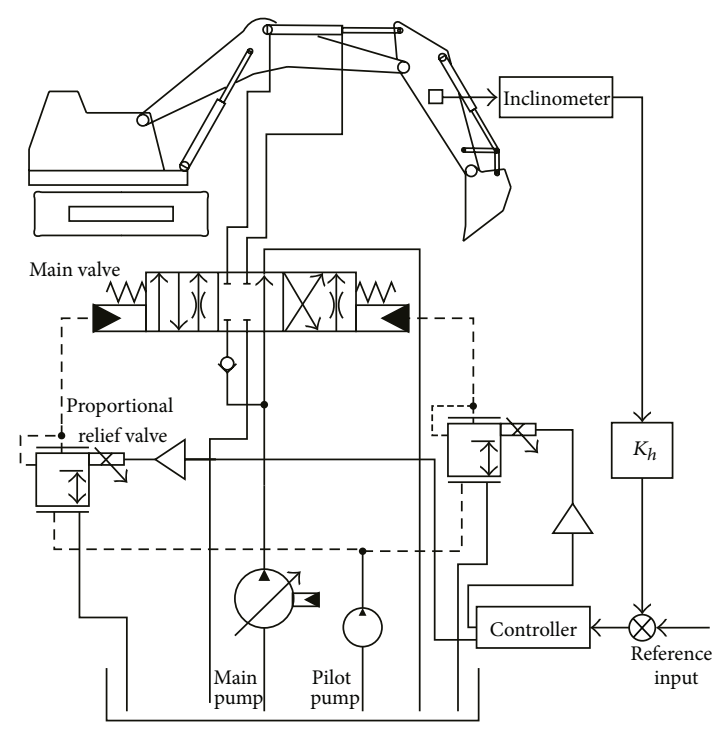

(b)

FIGURE 5: Experimental prototype machine.

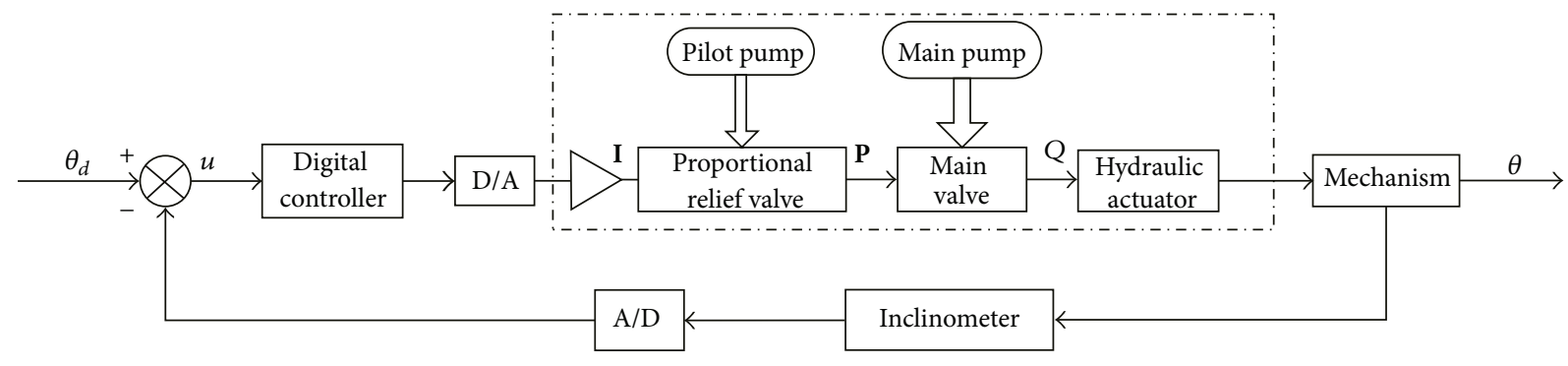

FIGURE 6: Electrohydraulic position servocontrol system.

In conclusion, the iterative identification algorithm can be presented as follwos.

Step 1. Set the initial values of $x(0), w(0), u(0)$, and $\mathbf{P}(0)$.

Step 2. Estimate the parameter $\widehat{\theta}(k)$ by algorithm (23) and calculate $\mathbf{P}(k)$ by $(24)$.

Step 3. Estimate the internal variables $\widehat{w}(k)$ and $\widehat{x}(k)$ by (25) and (26) using the recent estimates of model parameters $\hat{\theta}(k)$.

Step 4. Update the values of $\widehat{\Phi}(k)$ by (27).

Step 5. Return to Step 2 until the parameter estimates converge to constant values.

\section{Experiment}

5.1. Experimental Environment. A hydraulic excavator was retrofitted to be controlled by computer in our laboratory [23]. Figure 5 shows the prototype machine, whose manual pilot hydraulic control system was replaced by electrohydraulic proportional control system; inclinometers and pressure transducers were also installed on the excavator arms for position and force servocontrol. Schematic diagram of the electrohydraulic servosystem is shown in Figure 6.

5.2. Experimental Results. In order to obtain the nonlinear characteristics of the system when changing the directions and to obtain sufficient excitation, we adopted a multisine input signal which contained the frequency of $0.05 \mathrm{~Hz}, 0.1 \mathrm{~Hz}$, $0.2 \mathrm{~Hz}, 0.4 \mathrm{~Hz}$, and $0.5 \mathrm{~Hz}$ to the identification experiments. The sample rate was chosen to be $20 \mathrm{~Hz}$ on the machine. The input signal and angle output were obtained from the computer of the experiment machine. Ten groups of input and output signals with time duration of 55 seconds were sampled in the repeated experiments; the averaged measurement results are shown in Figure 7. Finally, we calculate the output angle velocity by numerical differentiation.

We set the parameters $n_{a}=3, n_{b}=2, n_{k}=8, r_{1}=r_{2}=3$, $x(0)=0, w(0)=0, u(0)=0$, and $\mathbf{P}(0)=10^{6} \mathbf{I}$. Note that lower forgetting factor $\lambda$ is useful for reducing the influences of old date, while a value of $\lambda$ close to 1 is less sensitive to disturbance. Therefore, we chose the forgetting factor to be $\lambda=0.98$ during the first 200 samples, and $\lambda=1$ otherwise. Compiling the developed iterative least square algorithm in MATLAB to identify the ARX model containing only the $L(z)$ 


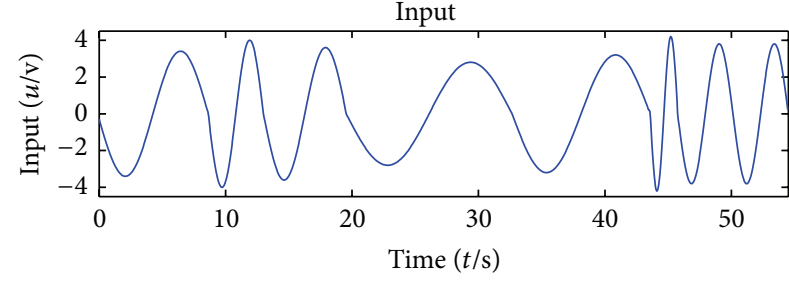

(a)

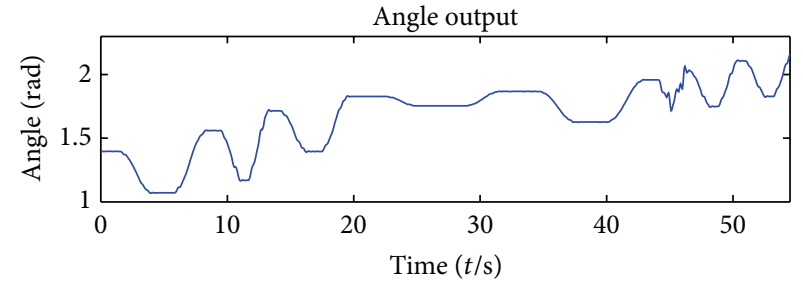

(b)

FIGURE 7: Input and output signals of the identification experiment.

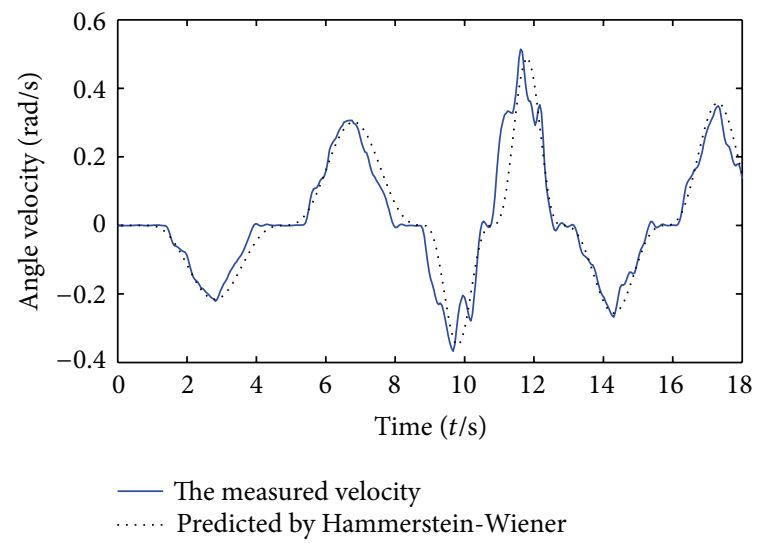

(a) Hammerstein-Wiener prediction result

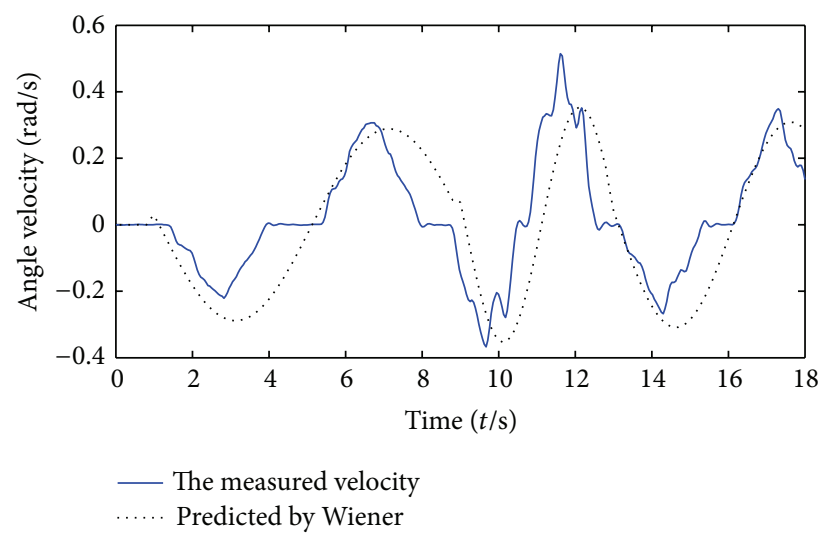

(c) Wiener prediction result

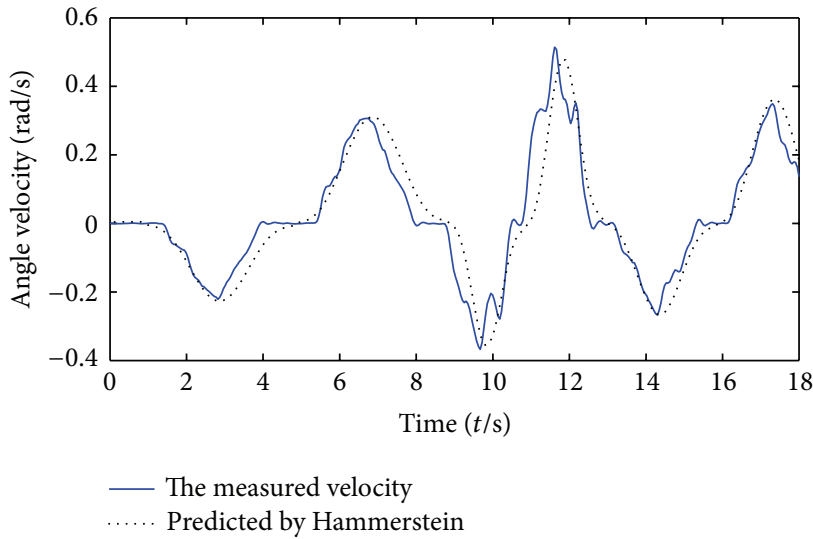

(b) Hammerstein prediction result

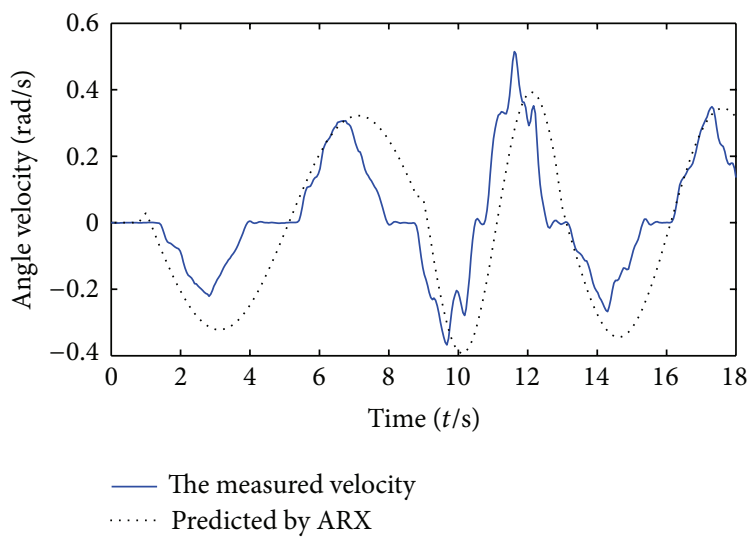

(d) ARX prediction result

Figure 8: Comparison results of the identified models.

block, Hammerstein model consisting of $N_{1}$ and $L(z)$, Wiener model consisting of $L(z)$ and $N_{2}$, and $\mathrm{H}-\mathrm{W}$ model consisting of $N_{1}, L(z)$, and $N_{2}$, respectively, we obtain the identification results shown in Table 1 .

We use the identified models to predict the tracking velocity of a general trajectory. The comparative results are shown in Figures 8 and 9. They demonstrate that the H-W and Hammerstein models which contain the input nonlinear block with two-segment polynomial nonlinearities capture the actual system well, while the Wiener and ARX models cannot approximate the actual system well. The mean-square errors (MSE) of the identified models in Table 2 show that the prediction error of the $\mathrm{H}-\mathrm{W}$ model is about $13 \%, 54 \%$, and $58 \%$ less than the Hammerstein, Wiener, and ARX models, respectively.

\section{Conclusion}

This paper investigates the nonlinear modeling and identification of an electrohydraulic control system. We develop a theoretic state space model for system analysis, propose an $\mathrm{H}$ W model for the highly nonlinear system based on a deeply physical insight into the actual system, and apply a modified 


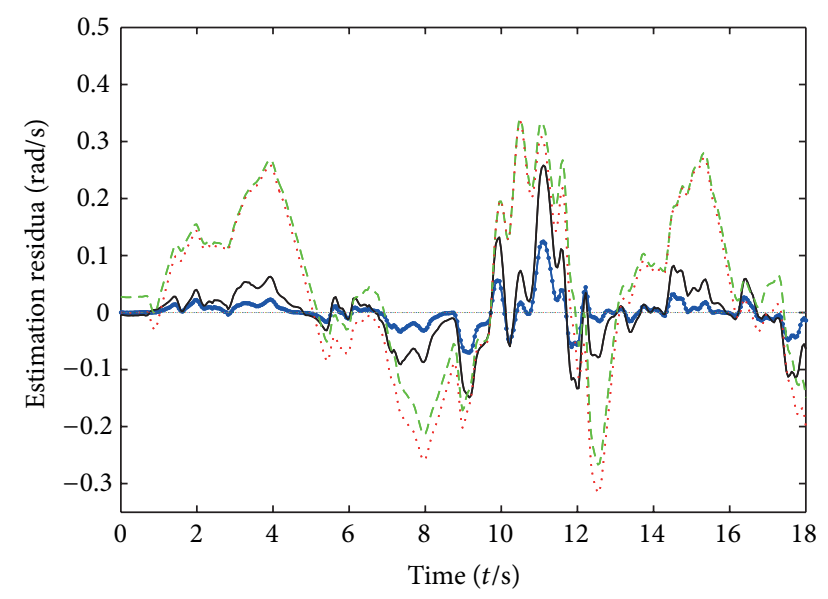

$\begin{array}{lll}\longrightarrow \text { Residua of Hammerstein-Wiener }--- & \text { Residua of Weiner } \\ \longrightarrow \text { Residua of Hammerstein } & \ldots . . & \text { Residua of ARX }\end{array}$

FIgURE 9: Comparison of the estimation residua.

TABLE 1: The identification results.

\begin{tabular}{|c|c|c|c|c|}
\hline \multirow{2}{*}{ Parameters } & \multicolumn{4}{|c|}{ Model type } \\
\hline & $\mathrm{H}-\mathrm{W}$ & Hammerstein & Wiener & ARX \\
\hline$b_{0}$ & 1 & 1 & -0.0358 & -0.0276 \\
\hline$b_{1}$ & 0.0349 & 0.0105 & 0.0151 & 0.0124 \\
\hline$b_{2}$ & 0.0617 & 0.0147 & -0.0069 & 0.0514 \\
\hline$a_{1}$ & -0.0309 & -0.0596 & 0.0069 & -0.0661 \\
\hline$a_{2}$ & -0.0598 & -0.0613 & -0.0075 & -0.0653 \\
\hline$a_{3}$ & -0.0897 & -0.0610 & -0.0081 & -0.0619 \\
\hline$f_{0}$ & 0.0109 & 0.0027 & - & - \\
\hline$f_{1}$ & $-1.4619 \times 10^{-5}$ & 0.0012 & - & - \\
\hline$f_{2}$ & -0.0050 & -0.0012 & - & - \\
\hline$f_{3}$ & 0.0052 & 0.0045 & - & - \\
\hline$p_{0}$ & -0.0165 & -0.0055 & - & - \\
\hline$p_{1}$ & 0.0033 & 0.0013 & - & - \\
\hline$p_{2}$ & 0.0104 & 0.0036 & - & - \\
\hline$p_{3}$ & -0.0010 & $-8.6858 \times 10^{-4}$ & - & - \\
\hline$q_{1}$ & 1 & - & 1 & - \\
\hline$q_{2}$ & 0.0050 & - & 0.0013 & - \\
\hline$q_{3}$ & $6.3562 \times 10^{-4}$ & - & $2.6895 \times 10^{-4}$ & - \\
\hline
\end{tabular}

TABLE 2: MSE of the identified models.

\begin{tabular}{lcccc}
\hline \multirow{2}{*}{ Errors } & \multicolumn{4}{c}{ Model type } \\
& $\mathrm{H}-\mathrm{W}$ & Hammerstein & Wiener & ARX \\
\hline$\delta / \mathrm{r} \cdot \mathrm{s}^{-1}$ & 0.0321 & 0.0417 & 0.1081 & 0.1218 \\
\hline
\end{tabular}

recursive least square method with internal variables estimation to identify its parameters. The main findings of the paper include the follwoing: (1) the proposed H-W model simplifies the identification procedure because it only uses the input and output signals to identify all the parameters. (2) The $\mathrm{H}-\mathrm{W}$ model containing the input nonlinear block with twosegment polynomial nonlinearities captures the actual system very well. As shown by the comparative results, the prediction error of the H-W model is about $13 \%, 54 \%$, and $58 \%$ less than the Hammerstein, Wiener, and ARX models, respectively. The results provide a physical insight into the nonlinear characteristics of the actual system, which is important for system analyzing, monitoring, and diagnosis. Future work includes addressing uncertain and fuzzy properties of the system $[24,25]$ and extending the model for a wider range of equipment [26].

\section{Acknowledgment}

This work was supported in part by grants from National Natural Science Foundation (Grant nos. 51175511 and 61105073) of China.

\section{References}

[1] H. E. Merritt, Hydraulic Control System, John Wiley \& Sons, New York, USA, 1967.

[2] B. Yao, F. Bu, J. Reedy, and G. T. C. Chiu, "Adaptive robust motion control of single-rod hydraulic actuators: theory and experiments," IEEE/ASME Transactions on Mechatronics, vol. 5, no. 1, pp. 79-91, 2000.

[3] S. Wen, W. Zheng, J. Zhu, X. Li, and S. Chen, "Elman fuzzy adaptive control for obstacle avoidance of mobile robots using hybrid force/position incorporation," IEEE Transactions on Systems, Man and Cybernetics C, vol. 42, no. 4, pp. 603-608, 2012.

[4] S. Y. Chen, J. Zhang, H. Zhang, N. M. Kwok, and Y. F. Li, "Intelligent lighting control for vision-based robotic manipulation," IEEE Transactions on Industrial Electronics, vol. 59, no. 8, pp. 3254-3263, 2012.

[5] Q. H. He, P. Hao, and D. Q. Zhang, "Modeling and parameter estimation for hydraulic system of excavator's arm," Journal of Central South University of Technology, vol. 15, no. 3, pp. 382386, 2008.

[6] K. Ziaei and N. Sepehri, "Modeling and identification of electrohydraulic servos," Mechatronics, vol. 10, no. 7, pp. 761$772,2000$.

[7] L. Wang, B. Wu, R. Du, and S. Yang, "Nonlinear dynamic characteristics of moving hydraulic cylinder," Chinese Journal of Mechanical Engineering, vol. 43, no. 12, pp. 12-19, 2007.

[8] M. Jelali and H. Schwarz, "Nonlinear identification of hydraulic servo-drive systems," IEEE Control Systems Magazine, vol. 15, no. 5, pp. 17-22, 1995.

[9] S. Kleinsteuber and N. Sepehri, "A polynomial network modeling approach to a class of large-scale hydraulic systems," Computers and Electrical Engineering, vol. 22, no. 2, pp. 151-168, 1996.

[10] H. Yousefi, H. Handroos, and A. Soleymani, "Application of differential evolution in system identification of a servo-hydraulic system with a flexible load," Mechatronics, vol. 18, no. 9, pp. 513528, 2008.

[11] F. Giri and E. W. Bai, Block-Oriented Nonlinear System Identification, vol. 404 of Lecture Notes in Control and Information Sciences, Springer, Berlin, Germany, 2010.

[12] B. -J. Kwak, A. E. Yagle, and J. A. Levitt, "Nonlinear system identification of hydraulic actuator friction dynamics using a Hammerstein model," in Proceedings of the IEEE International Conference on Acoustics, Speech and Signal Processing, vol. 4, pp. 1933-1936, Seattle, Wash, USA, 1998. 
[13] Y. J. Zheng, S. Y. Chen, Y. Lin, and W. L. Wang, "BioInspired optimization of sustainable energy systems: a review," Mathematical Problems in Engineering, vol. 2013, Article ID 354523, 12 pages, 2013.

[14] C. Cattani, R. Badea, S. Chen, and M. Crisan, "Biomedical signal processing and modeling complexity of living systems," Computational and Mathematical Methods in Medicine, vol. 2012, Article ID 298634, 2 pages, 2012.

[15] B. Li, J. Yan, A. X. Liu, Y. H. Zeng, and G. Guo, "Nonlinear identification of excavator's electro-hydraulic servo system," Transactions of the Chinese Society For Agricultural Machinery, vol. 43, no. 4, pp. 20-25, 2012.

[16] J. Ljung, System Identification, Theory for the User, Prentice Hall Press, New York, NY, USA, 1999.

[17] S. Chen, W. Huang, C. Cattani, and G. Altieri, "Traffic dynamics on complex networks: a survey," Mathematical Problems in Engineering, vol. 2012, Article ID 732698, 23 pages, 2012.

[18] J. Vörös, "Iterative algorithm for parameter identification of Hammerstein systems with two-segment nonlinearities," IEEE Transactions on Automatic Control, vol. 44, no. 11, pp. 2145-2149, 1999.

[19] J. Vörös, "Recursive identification of Hammerstein systems with discontinuous nonlinearities containing dead-zones," IEEE Transactions on Automatic Control, vol. 48, no. 12, pp. 2203-2206, 2003.

[20] J. Vörös, "An iterative method for Hammerstein-Wiener systems parameter identification," Journal of Electrical Engineering, vol. 55, no. 11-12, pp. 328-331, 2004.

[21] J. Vörös, "Identification of nonlinear dynamic systems using extended Hammerstein and Wiener models," Control Theory and Advanced Technology, vol. 10, no. 4, part 2, pp. 1203-1212, 1995.

[22] E.-W. Bai, "A blind approach to the Hammerstein-Wiener model identification," Automatica, vol. 38, no. 6, pp. 967-979, 2002.

[23] J. Yan, B. Li, Q. Z. Tu, G. Gang, and Y. H. Zeng, "Automatization of excavator and study of its auto-control," in Proceedings of the 3rd International Conference on Measuring Technology and Mechatronics Automation, pp. 604-609, Shanghai, China, 2011.

[24] Y. J. Zheng and H. F. Ling, "Emergency transportation planning in disaster relief supply chain management: a cooperative fuzzy optimization approach," Soft Computing, 2013.

[25] Y. J. Zheng and S. Y. Chen, "Cooperative particle swarm optimization for multiobjective transportation planning," Applied Intelligence, 2013.

[26] Y. Zheng, S. Chen, and H. Ling, "Efficient multi-objective tabu search for emergency equipment maintenance scheduling in disaster rescue," Optimization Letters, vol. 7, no. 1, pp. 89-100, 2013. 


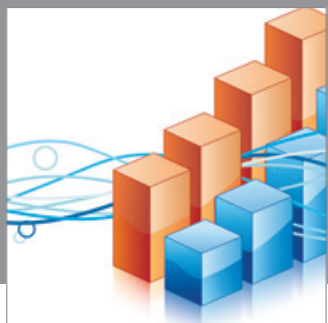

Advances in

Operations Research

mansans

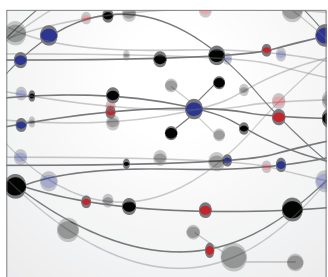

The Scientific World Journal
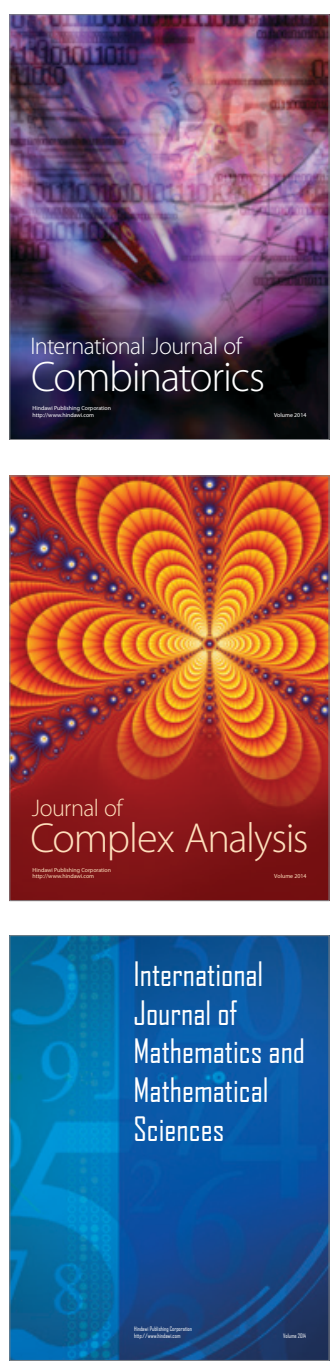
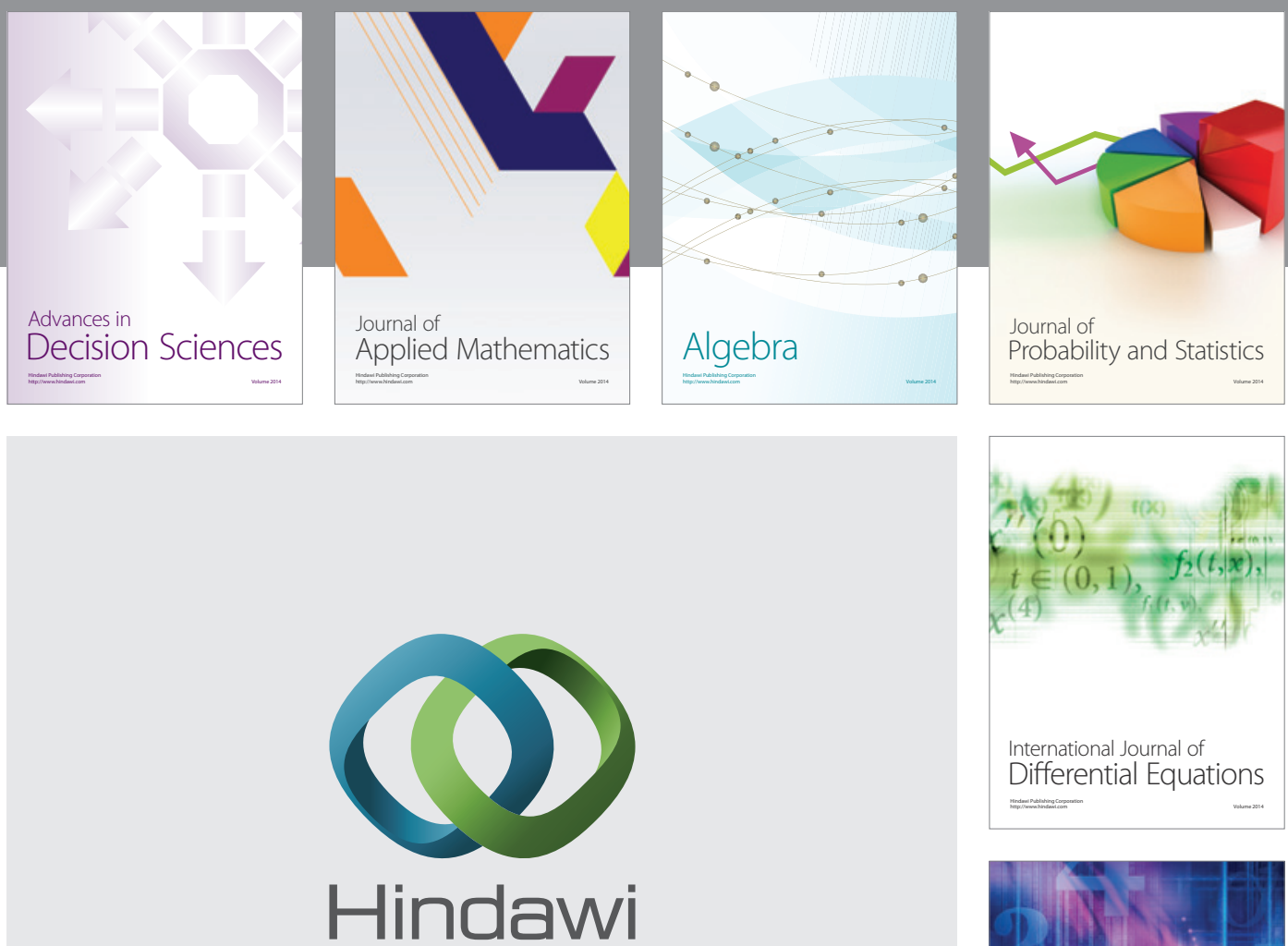

Submit your manuscripts at http://www.hindawi.com
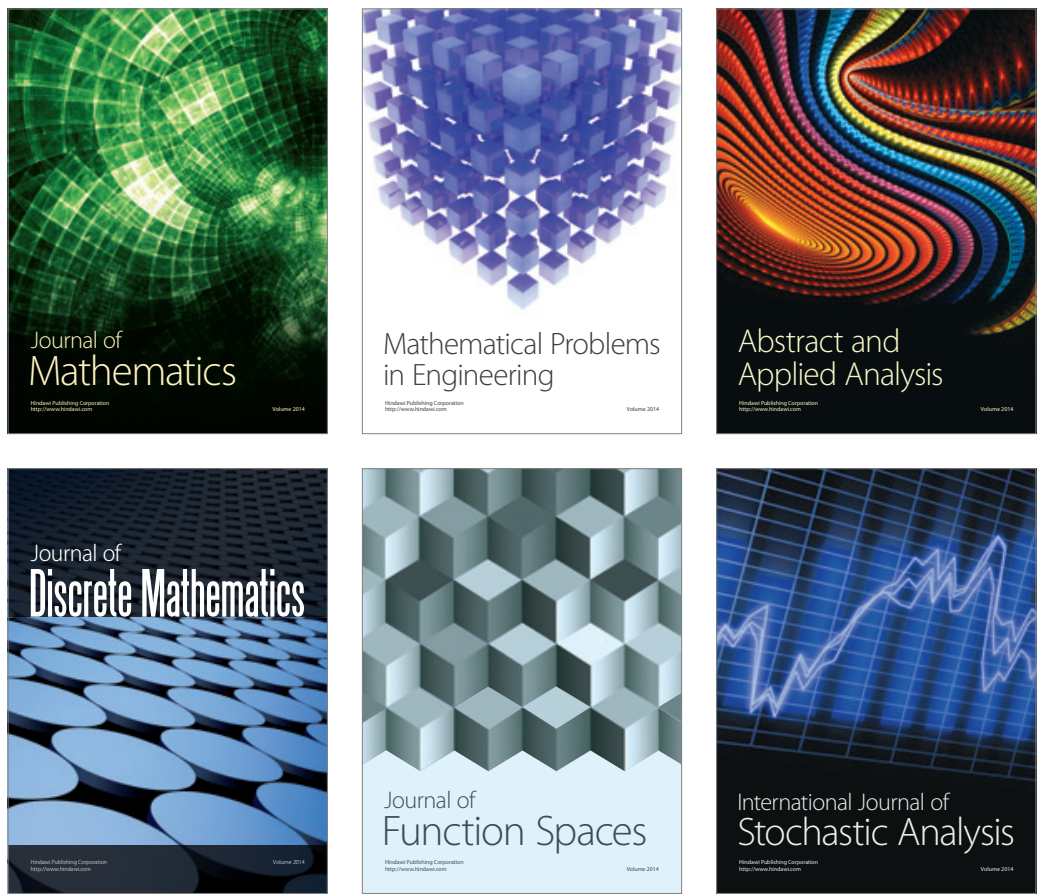

Journal of

Function Spaces

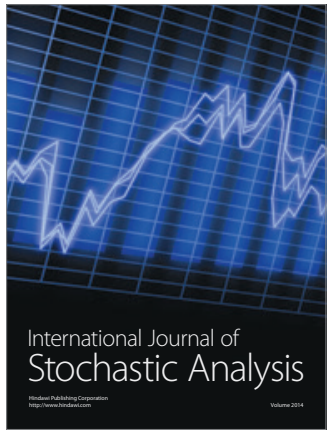

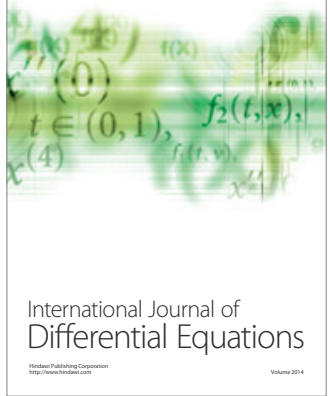
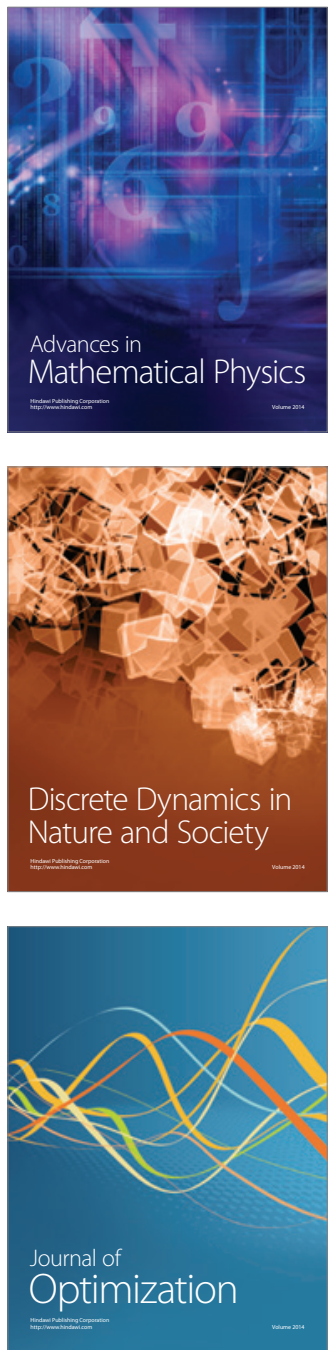